\title{
Efficacy of Intentional Permanent Atrial Pacing in the Long-Term Management of Congenital Long QT Syndrome
}

\author{
Gurukripa Kowlgi ${ }^{1}$, John Giudicessi ${ }^{1}$, Walid Barake ${ }^{1}$, Johan Bos ${ }^{1}$, and Michael Ackerman ${ }^{1}$ \\ ${ }^{1}$ Mayo Clinic
}

June 30, 2020

\begin{abstract}
Background: Some long QT syndrome (LQTS) patients experience breakthrough cardiac events (BCEs) despite maximal therapy. Small studies have shown that intentional permanent atrial pacing (IPAP) is beneficial in refractory LQTS. As such, we sought to determine the genotype-specific utilization and efficacy of IPAP in a single-center LQTS registry. Methods and Results: In this retrospective study, electronic medical records from 1,065 LQTS patients were used to identify individuals that received IPAP. Pre- and post-IPAP heart rate, heart rate-corrected QT (QTc) values, annual BCE rate, and IPAP-related complications were compared between genotypes. BCEs were defined as LQTS-associated syncope/seizures, ventricular arrhythmia (VA)terminating ICD therapies, and sudden cardiac arrest/death. Overall, 52/1065 LQTS patients received adjunctive IPAP therapy [77\% female; median age 18.5 (IQR 1-35.5) years; 73\% with prior VA]. Over an average IPAP follow-up of 121 ? 82 months, the average heart rate increased from $65.8 ? 20.4 \mathrm{bpm}$ to $78.9 ? 17.1 \mathrm{bpm} ;(\mathrm{p}<0.01)$ and the average QTc decreased from 533.4 ? $66.6 \mathrm{~ms}$ to 488.3 ? $52.4 \mathrm{~ms}$; $(\mathrm{p}<0.01)$. The mean BCE rate dropped from 0.88 to 0.19 per patient-year $(\mathrm{p}=0.01)$, driven by a marked decrease in LQT2 patients (1.01 BCE/year to $0.02 \mathrm{BCE} /$ year; $\mathrm{p}=0.003$ ). No serious IPAP-related complications were observed. Conclusion: In high-risk LQTS patients, namely those with recalcitrant LQT2, IPAP appears to be a safe and efficacious adjunct therapy. The beneficial effects of IPAP may stem from attenuating the QTc and circumventing a pause-dependent trigger. Whether IPAP might obviate the need for an ICD in some instances warrants further study.
\end{abstract}

\section{Hosted file}

2020 - JCE - IPAP in LQTS - MANUSCRIPT.docx available at https://authorea.com/users/ 338358/articles/464366-efficacy-of-intentional-permanent-atrial-pacing-in-the-long-

term-management-of-congenital-long-qt-syndrome 